Basic Science

\title{
Rapid and Delayed Effects of Pulsed Radiofrequency on Neuropathic Pain: Electrophysiological, Molecular, and Behavioral Evidence Supporting Long-Term Depression
}

\author{
Ren-Yu Huang, MS', Chia-Chi Liao, PhD², Shih-Ying Tsai, $\mathrm{PhD}^{3}$, Chen-Tung Yen, $\mathrm{PhD}^{4}$, \\ Chii-Wann Lin, PhD ${ }^{5}$, Tsung-Chi Chen, MS ${ }^{5}$, Wei-Tso Lin, MS ${ }^{6}$, Chi-Heng Chang ${ }^{5}$, and \\ Yeong-Ray Wen, MD, $\mathrm{PhD}^{3,7-9}$
}

From: ${ }^{1}$ Graduate Institute of Clinical Medical Science, China Medical University, Taichung, Taiwan; ${ }^{2}$ Central lab, Shin-Kong Wu Ho-Su Memorial Hospital, Taipei, Taiwan; ${ }^{3}$ Department of Anesthesiology, School of Medicine, China Medical University, Taichung, Taiwan; Institute of Zoology, National Taipei University, Taipei,

Taiwan; 5nstitute of Biomedical Engineering, National Taiwan University, Taipei, Taiwan; ${ }^{6}$ Institute of Biomedical Electronics and Bioinformatics, National Taiwan University, Taipei, Taiwan; ${ }^{7} \mathrm{G}$ raduate Institute of Acupuncture Science, College of Chinese Medicine, China Medical University, Taichung, Taiwan; ${ }^{8}$ Pain Center, Department of Anesthesiology, China Medical University Hospital, Taichung, Taiwan;9 Acupuncture Research Center, China Medical University, Taichung, Taiwan

Address Correspondence: Yeong-Ray Wen, MD, PhD Center for Pain Management and Research, Department of Anesthesiology, China Medical University Hospital, No. 2, YuhDer Rd, North District, 40447, Taichung, Taiwan E-mail:yr.wen@yahoo.com.tw; yrwen@mail.cmu.edu.tw

Disclaimer on P. E281

Manuscript received: 03-13-2016 Revised manuscript received: 07-27-2016 Accepted for publication: 08-03-2016

Free full manuscript: www.painphysicianjournal.com
Background: Pulsed radiofrequency (PRF) has been widely employed for ameliorating clinical neuropathic pain. How PRF alters electrophysiological transmission and modulates biomolecular functions in neural tissues has yet to be clarified. We previously demonstrated that an early application of low-voltage bipolar PRF adjacent to the dorsal root ganglion (DRG) reduced acute neuropathic pain in animals. By contrast, the present study investigated how PRF alters postsynaptic sensitization to produce early and delayed effects on neuropathic pain

Objectives: Our objective was to test the hypothesis that a 5-minute session of PRF could rapidly produce selective long-term depression (LTD) on C-fiber-mediated spinal sensitization and sustain the effect through the long-lasting inhibition of injury-induced ERK-MAPK activation. This may explain the prolonged analgesic effect of PRF on chronic neuropathic pain.

Study Design: Experiments were conducted on both normal rats and neuropathic pain rats that received spinal nerve ligation (SNL) 8 days prior.

Setting: An animal laboratory in a medical center of a university in Taiwan.

Methods: We first compared changes in field potentials in the L5 superficial spinal dorsal horn $(\mathrm{SDH})$ that were evoked by conditioning electrical stimuli in the sciatic nerve in male adult rats before (as the baseline) and after PRF stimulation for at least 2 hours. Bipolar PRF was applied adjacent to the L5 DRG at an intensity of $5 \mathrm{~V}$ for 5 minutes, whereas the control rats were treated with sham applications. The electrophysiological findings were tested for any correlation with induction of spinal phospho-ERK (p-ERK) in normal and neuropathic pain rats.

We then investigated the delayed effect of PRF on SNL-maintained pain behaviors for 2 weeks as well as p-ERK in SDH among the control, SNL, and PRF groups. Finally, potential injury in the DRGs after PRF stimulation was evaluated through behavioral observations and ATF-3, a neuronal stress marker.

Results: In the evoked field-potential study, the recordings mediated through A- and C-afferent fibers were identified as A-component and C-component, respectively. PRF significantly reduced the C-components over 2 hours in both the normal and SNL rats, but it did not affect the A-components. In the SNL rats, the C-component was significantly depressed in the PRF group compared with the sham group. PRF also inhibited acute $\mathrm{p}$-ERK induced by mechanical nociception in both the control and SNL rats.

For a longer period, PRF ameliorated SNL-maintained mechanical allodynia for 10 days and thermal analgesia for 14 days, and it significantly reduced late ERK activation within spinal neurons and astrocytes 14 days afterward. Moreover, PRF in the normal rats did not alter basal withdrawal thresholds or increase the expression and distribution of ATF-3 in the DRGs.

Limitations: Several issues should be considered before translating the animal results to clinical applications. 
Conclusion: Low-voltage bipolar PRF produces LTD through selective suppression on the C-component, but not on the A-component. It also inhibits ERK activation within neurons and astrocytes in SDHs. The findings suggest that PRF alleviates long-lasting neuropathic pain by selectively and persistently modulating C-fiber-mediated spinal nociceptive hypersensitivity.

Key words: Pulsed radiofrequency (PRF), dorsal root ganglion (DRG), neuropathic pain, ERK activation, evoked field potential, ATF-3, long-term depression (LTD), spinal nerve ligation (SNL)

Pain Physician 2017; 20:E269-E283

P ulsed radiofrequency (PRF) has been widely applied adjacent to the dorsal root ganglion (DRG) or peripheral nerves for chronic neuropathic pain diseases, such as lumbar or cervical radicular pain, postherpetic neuralgia, and various peripheral neuralgia conditions (1-4). In contrast to the destructive effect of conventional RF (CRF), PRF causes little to no neural damage $(5,6)$. PRF is typically believed to modulate the pain pathology without impairing motor functions or innocuous sensations (1,2,6-11). Nevertheless, evidence on so-called neuromodulation remains scant.

Earlier morphological and molecular studies have presented some implications. Under a light microscope, both myelinated $\mathrm{A}$ - and unmyelinated $\mathrm{C}$-fiber neurons within DRGs have been found to be unchanged after PRF application $(10,12)$. However, studies using electron microscopy revealed that PRF caused ultrastructural changes in DRG neurons, but conflicting results were shown (10,12-15). For example, subcellular organelles of large ganglionic cells and myelinated axons had been advocated to be preferentially damaged to a greater extent compared with small DRG cells and unmyelinated axons $(12,13,15)$, but others indicated that PRF engendered greater damage to $\mathrm{C}$-fiber than to $\mathrm{A}$-fiber axons within the sciatic nerve (14). Recent studies have confirmed that PRF regulated intracellular signaling and synaptic plasticity in the spinal dorsal horns (SDH) $(4,8,11)$, such as increased Fos expression (16), reduced presynaptic release of glutamate and citrulline (17), and the induction of supraspinal descending inhibitory pathways through 5-HT3 and $\alpha 2$ receptors (18). Therefore, it becomes important to link these fragments of knowledge to elucidate PRF's mechanisms.

Long-term potentiation (LTP), an increase in postsynaptic currents or potentials evoked by a single monosynaptic preganglionic action potential, is involved throughout nociceptive sensitizing processes (19). Such synaptic upregulations in the SDH can be induced by natural stimuli such as inflammation and nerve injury, or a conditioning stimulus such as peripheral electric pulses $(20,21)$. Accumulating evidence indicated that 2 LTP phases, early (minutes to hours) and late (days to weeks), control a myriad of sensitizing molecules in the central nervous system for maintaining and enhancing pathological pain $(20,22,23)$. In particular, distinct signals such as phospho-ERK ( $p$-ERK) and Brain-derived neurotropic factor (BDNF) $(24,25)$ are directly involved in the transition from acute to late phase of LTP (20). Therefore, amelioration of LTP can eliminate acute hyperalgesia and erase trace memories of pain $(19,26)$. We previously demonstrated that early PRF intervention inhibited spinal p-ERK, p-p38, and TNF $\alpha$ at the acute post-nerve injury stage (27). Therefore, our next objective is to determine how PRF alters synaptic plasticity, pain behaviors, and neuronal-glial interactions.

By recording evoked-field potentials, effects of PRF on different types of afferent fibers could be identified. Moreover, we examined nocifensive behaviors and investigated $p$-ERK changes over time. Finally, potential stresses of DRG cells from PRF stimulation were evaluated. These findings may enhance our understanding of the complex neuromodulatory actions of PRF.

\section{Methods}

\section{Animal Preparation}

Experiments were performed on adult male Sprague-Dawley rats (220 - 250 g; BioLASCO, Taiwan). The animals were housed in groups of 2 to 3 in plastic cages at a constant temperature of $22{ }^{\circ} \mathrm{C}$ and at a relative humidity of $40 \%-60 \%$. They were fed food and water ad libitum in a 12-hour light/dark cycle environment for at least 5 days before the experiments.

The study protocol was approved by the Institutional Animal Care and Utilization Committee, China Medical University Hospital, Taichung, Taiwan. All experiments were conducted in accordance with the "Ethical Guidelines for the Treatment of Animals" by the International Association for the Study of Pain (28). 
The normal rats in the following experiments indicated the rats which did not received any surgery before PRF stimulation, and should be distinct from those rats in the control groups which received sham SNL surgery.

\section{Specially Designed PRF Device for Animal DRG Application}

The specially designed bipolar system has been described previously $(27,29)$. The stimulation electrode was inserted into the left L5 foraminal canal, whereas the reference electrode was placed in contact with the surrounding non-neural tissues. The electrodes were connected to a PXI-5402 Function Generator (National Instruments, Austin, TX) to generate RF pulses with the following parameter settings based on clinical settings: 2-Hz biphasic trains with $500-\mathrm{kHz}$ RF waves, 25-ms train width, and oscillating amplitudes at an intensity of \pm 2.5 V. The PRF duration was 300 seconds. The control group received an electrode placement without electricity as a sham stimulation.

\section{Spinal Nerve Ligation}

The spinal nerve ligation (SNL) surgery was performed in accordance with our laboratory protocol $(27,29)$ on Day 0 . A deep skin incision was made between the left iliac crest and the lower lumbar spine while the animal was anesthetized with $2 \%$ isoflurane. The L5 spinal nerve was exposed and tightly ligated with a 6-0 silk thread $(30,31)$. PRF stimulation was performed on Day 8.

\section{Evoked-field Potential (EP) Recordings}

Under $2 \%$ isoflurane anesthesia, the animals were stereotaxically mounted, artificially ventilated, and paralyzed with gallamine ( $20 \mathrm{mg} / \mathrm{kg}$, i.v., every 2 hours). The body temperature was maintained at $37^{\circ} \mathrm{C}$. The left sciatic nerve was exposed at the midhind thigh level and stimulated with a bipolar silver hook electrode as a conditioning stimulus. The L5 segment was exposed after a laminectomy for the penetration of a tungsten microelectrode ( $5 \mathrm{M} \Omega$; $\mathrm{A}-\mathrm{M}$ system, Olympic Peninsula, WA). While the single monophasic electricity stimulated the sciatic nerve $(2 \mathrm{~Hz}, 3 \mathrm{~mA}, 0.5 \mathrm{~ms})$, the EPs were recorded at a depth of $100-300 \mu \mathrm{m}$ in the superficial SDH and signals were analyzed using the PowerLab system (AD Instrument, Sydney, Australia). For each recording trace, we defined the first negative potential (P1) appearing between 0 and $90 \mathrm{~ms}$ as the A-component, and the second negative potential (P2) between 90 and 300 ms as the C-component (Fig. 1). The recording site was determined when the C-component was detected consistently with conditioning stimulus at a $2.5 \times \mathrm{C}$-fiber threshold (typically 5-10 mA).

The peripheral stimulation was repeated every 100 seconds to avoid neuronal habituation or sensitization. At least 18 consecutive stable $\mathrm{A}$ - and C-components were recorded (i.e., 30 minutes) before PRF/sham stimulation, and were averaged as a baseline response. Ten minutes after the 300-second PRF/sham stimulation, EP recordings were re-executed continuously for at least 2.5 hours. Every 6 post-PRF EPs (10-minute intervals) were averaged and defined as test responses. The magnitudes of the evoked A-/C-components were calculated by integrating the area within the negative potential, and they were standardized to the individual baseline value of each rat. Therefore, the PRF effects were the response ratio by using the equation: (test response - baseline response)/baseline response $\times 100 \%$. After the experiment, the recording site was marked with an electrolytic lesion, and the spinal cord was removed for Nissl staining to confirm the recording sites.

\section{Nociceptive Behaviors}

The mechanical threshold was evaluated using von Frey filaments (Stoelting, Wood Dale, IL) with the up-down method (32), and it was calculated using a $50 \%$ withdrawal threshold (33). In brief, the left hind paw of each animal placed in a chamber of a Plexiglas cage was pressed using one of 8 von Frey fibers with incremental strength $(0.4-26.0 \mathrm{~g})$ onto the plantar surface. The tests were conducted daily from at least 2 days before SNL for establishing preoperative baselines, and scheduled days after SNL as well as after PRF/ sham treatment.

The thermal threshold was measured by paw withdrawal latencies to radiant heat stimulation in the plantar test device (Plantar Test Apparatus, IITC, CA). The cut-off latency was 30 seconds to avoid thermal injury. The withdrawal latency at each time point was an average of 3 latencies separated by a 5-minute interval. The tests were conducted on the same days as the von Frey test and both tests were conducted by the same experimenter (Huang, RY) who was blinded to the group allocation.

\section{Immunostaining Analysis}

The animals were deeply anesthetized with isoflurane and transcardially perfused with $37^{\circ} \mathrm{C}$ saline, followed by $4 \%$ paraformaldehyde. The lower lumbar 


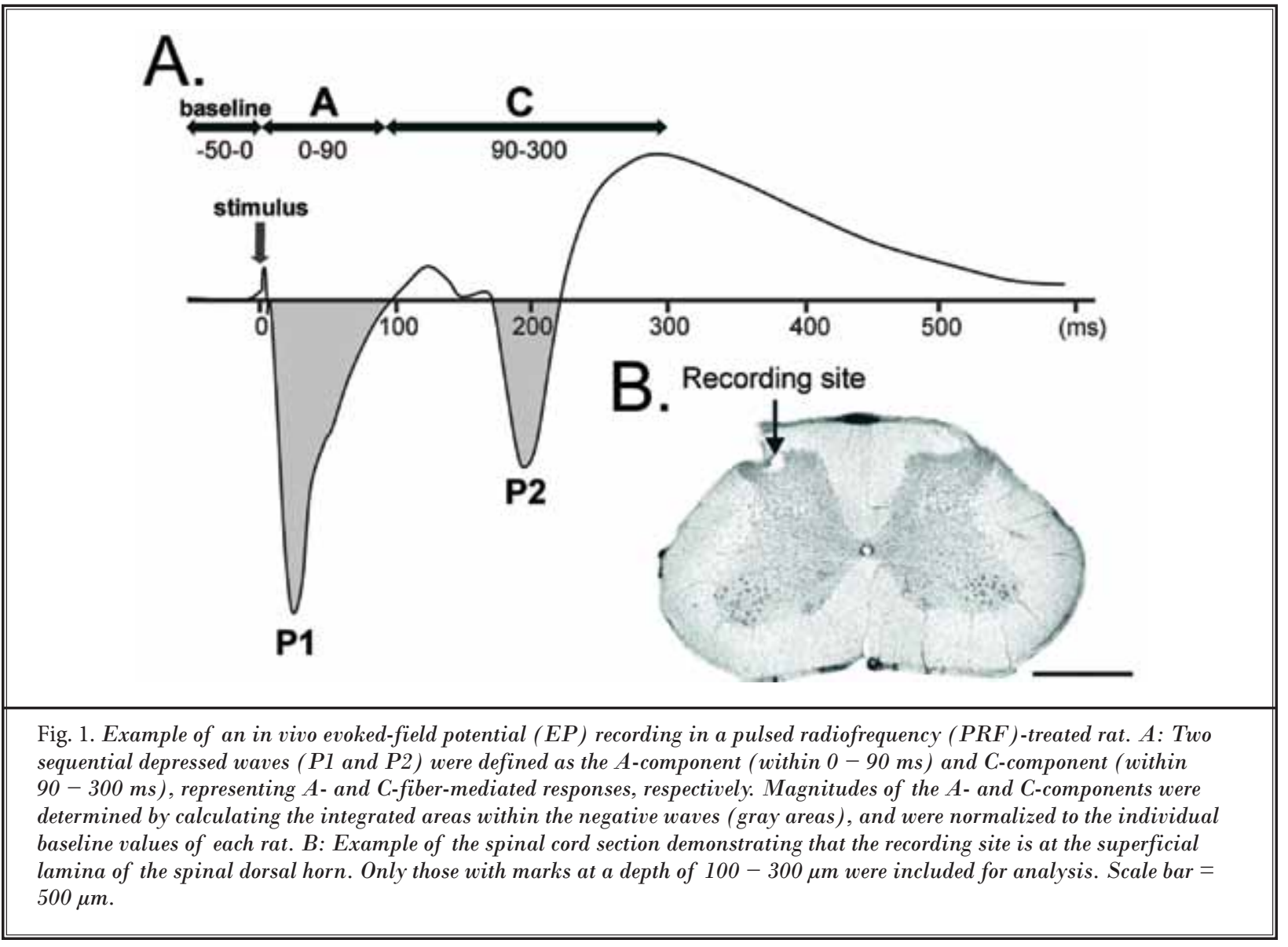

spinal cord and bilateral L4 and L5 DRGs were removed, post-fixed, and cryoprotected. The spinal cord and DRG were cut in a cryostat at thicknesses of 30 and $10 \mu \mathrm{m}$, respectively. The spinal cord sections were incubated with a mixture of anti-p-ERK (1:400; Cell Signaling Technology, Danvers, MA) and anti-NeuN (neuron marker, 1:1500; Chemicon, Temecula, CA), anti-OX42 (microglia marker, 1:200; Serotec, Indianapolis, IN), or anti-GFAP (astrocyte marker, 1:6000; Chemicon) antibodies, followed by incubation with a mixture of Alexa 488- and Cy3-conjugated secondary antibodies (Jackson ImmunoResearch, West Grove, PA). The DRG sections were blocked and incubated with an anti-ATF-3 antibody (1:500, Sigma, San Louis, MO) overnight and were reacted with biotinylated goat anti-rabbit IgG (1:200, Vector Laboratories, Burlingame, $C A$ ) by using the $A B C$ method (Elite $A B C$ kit, Vector Laboratories). Images of all section were captured using a CCD camera connected to a Zeiss Axio Imager A2 microscope (Göttingen, Germany). At a magnification of 20x, neucleated immunoreactive (ir) cells were counted by laminae (I-V) from randomly chosen sections (DP Controlled, 3.3.1, Olympus), at least 6 sections from each DRG or lumbar segment, and the numbers were averaged to represent segmental expressions. Negative control staining was conducted by omitting the primary antibody.

\section{Study Design}

\section{Experiment 1: Rapid effect of PRF on EPs in normal (un-injured) rats}

The normal rats were allocated to 2 groups, for PRF or sham stimulation. In the sham group, the PRF electrode was placed without electric current. EP recordings were performed before and after PRF/sham stimulation (Table 1).

\section{Experiment 2: Rapid effect of PRF on EPs in SNL rats}

Eight days after SNL, the nerve-ligated rats were allocated to the PRF (SNL+PRF) or sham (SNL+sham) group for recording. 
Table 1. Study design.

\begin{tabular}{|c|c|c|c|c|c|c|c|c|}
\hline$\overline{E x p}$ & Design & $\overline{\text { Groups }}$ & 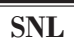 & MS & PRF & PRF Effect & Rat \# & Figure \# \\
\hline 1 & EP study & $\begin{array}{l}\text { Sham } \\
\text { PRF }\end{array}$ & - & NA & $\begin{array}{l}- \\
+\end{array}$ & Early & $\begin{array}{l}6 \\
6\end{array}$ & 2 \\
\hline 2 & EP study & $\begin{array}{l}\text { SNL+Sham } \\
\text { SNL+PRF }\end{array}$ & $\begin{array}{l}+ \\
+\end{array}$ & NA & $\begin{array}{l}- \\
+\end{array}$ & Early & $\begin{array}{l}6 \\
6\end{array}$ & 3 \\
\hline 3 & p-ERK & $\begin{array}{c}\text { C+Sham } \\
\text { C+PRF } \\
\text { C+MS+Sham } \\
\text { C+MS+PRF }\end{array}$ & $\begin{array}{l}- \\
- \\
- \\
-\end{array}$ & $\begin{array}{l}- \\
- \\
+ \\
+\end{array}$ & $\begin{array}{l}- \\
+ \\
- \\
+\end{array}$ & Early & $\begin{array}{l}5 \\
4 \\
4 \\
4\end{array}$ & $4 \mathrm{~A}, 4 \mathrm{~B}$ \\
\hline 3 & p-ERK & $\begin{array}{c}\text { SNL+Sham } \\
\text { SNL+MS+Sham } \\
\text { SNL+MS+PRF }\end{array}$ & $\begin{array}{l}+ \\
+ \\
+\end{array}$ & $\begin{array}{l}- \\
+ \\
+\end{array}$ & $\begin{array}{l}- \\
- \\
+\end{array}$ & Early & $\begin{array}{l}4 \\
4 \\
4\end{array}$ & $4 \mathrm{C}, 4 \mathrm{D}$ \\
\hline 4 & Behavior & $\begin{array}{c}\text { C+Sham } \\
\text { SNL+Sham } \\
\text { SNL+PRF }\end{array}$ & $\begin{array}{l}+ \\
+ \\
+\end{array}$ & NA & $\begin{array}{l}- \\
+\end{array}$ & Delayed & $\begin{array}{l}8 \\
8 \\
9\end{array}$ & 5 \\
\hline 5 & p-ERK & $\begin{array}{c}\text { C+Sham } \\
\text { SNL+Sham } \\
\text { SNL+PRF }\end{array}$ & $\begin{array}{l}- \\
+ \\
+\end{array}$ & NA & $\begin{array}{l}- \\
+\end{array}$ & Delayed & $\begin{array}{l}4 \\
4 \\
4\end{array}$ & $\begin{array}{l}\text { 6A, 6B (1 day) } \\
\text { 6C, 6D (14 day) }\end{array}$ \\
\hline 6 & Behavior & $\begin{array}{l}\text { Sham } \\
\text { PRF }\end{array}$ & - & NA & $\begin{array}{l}- \\
+\end{array}$ & Delayed & $\begin{array}{l}6 \\
8\end{array}$ & 7 \\
\hline 6 & ATF3 & $\begin{array}{l}\text { Sham } \\
\text { Sham-E } \\
\text { PRF }\end{array}$ & $\begin{array}{l}- \\
- \\
-\end{array}$ & NA & $\begin{array}{l}- \\
- \\
+\end{array}$ & Delayed & $\begin{array}{l}5 \\
4 \\
4\end{array}$ & 8 \\
\hline
\end{tabular}

Exp: experiment number in the study design section (see text); C: control, EP: evoked potential, MS: noxious mechanical stimulation, NS: not applicable, PRF: pulsed radiofrequency, Sham-E: sham surgery for electrode placement without PRF, SNL: spinal nerve ligation; \#: number.

\section{Experiment 3: Rapid effect of PRF on intense noxious stimulation-evoked ERK activation in the control and SNL rats}

To determine the molecular responses in the SDH in Experiments 1 and 2, p-ERK induction by repetitive intense mechanical stimuli was analyzed in control and SNL rats with and without PRF stimulation, respectively. The left hind paw of the anesthetized rat was rapidly and strongly poked with a thick von Frey fiber (\#18, $50 \mathrm{~g})$ for 2 cycles of $20 \mathrm{x}$ applications within 10 seconds for each, with 10 seconds of separation. Therefore, 40 intense stimuli were applied. Similar suprathreshold stimulations have been reported to cause hyperalgesic responses and p-ERK expression (34,35). PRF was applied immediately after mechanical stimulation. The rats were sacrificed 30 minutes afterward for harvesting the L4-5 segments. The sham-PRF group was treated similarly, except for the PRF.

\section{Experiment 4: Delayed effect of PRF on pain behaviors in SNL rats}

There were 3 groups: the control (sham SNL) group with sham PRF (C+Sham) group, SNL+Sham group, and SNL+PRF group. Pain behaviors before and after SNL, and after PRF were compared.

\section{Experiment 5: Delayed effect of PRF on SNL- maintained p-ERK activities}

Because p-ERK could be time-dependently present in different spinal cells, its appearance over time is important. p-ERK were compared among 3 groups on post-PRF Day 1 and Day 14: the Control (C+Sham) group, SNL (SNL+Sham) group, and SNL+PRF group; moreover, cellular co-expression with $\mathrm{p}$-ERK staining were examined.

\section{Experiment 6: PRF effect on the treated DRG: behavioral and molecular changes}

This study aimed to ascertain if electrode insertion led to unexpected damages. First, mechanical thresholds were compared in the normal rats with and without PRF (PRF vs sham). Second, the expression of ATF-3, a neuronal stress marker, in the DRG was analyzed among 3 groups: rats with a back incision (Sham), with an incision for electrode placement (Sham-E), and with an incision plus PRF stimulation (PRF). Bilateral L4 and L5 DRGs were harvested 7 days after PRF/sham. The ATF-3 expression was analyzed by calculating the ratio of ATF3-ir neurons to total neurons ("positive-staining ratio") and by calculating the radius ratio of small, medium-sized, and large ATF3-ir cells (indicating C-, 


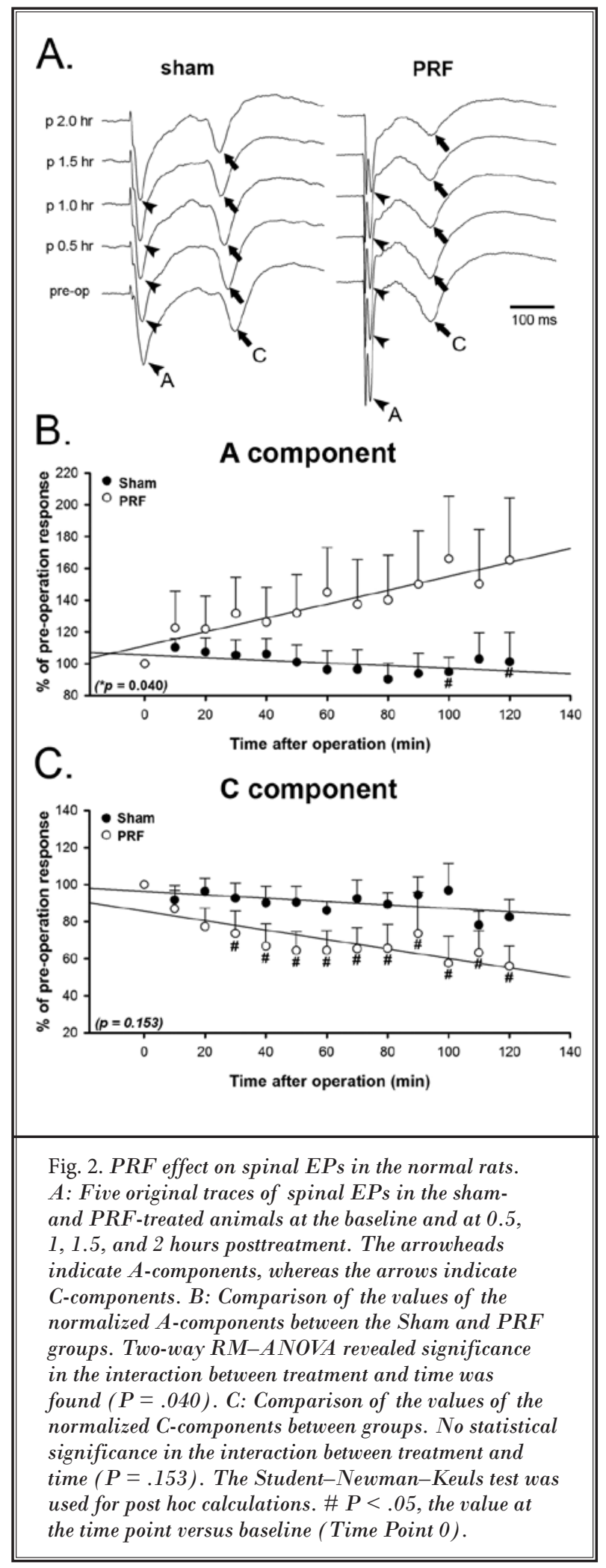

$A \delta$-, and $A \beta$-fiber neurons, respectively) in proportion to total ATF3-ir neurons ("cell-size distribution") by using NIH software (ImageJ, 1.4.7v) for cell counting and size calculation. This analytic method has been widely used (36-38).

\section{Statistical Analysis}

Data are presented as the mean \pm standard error of the mean (SEM). The effect of PRF on the A-/Ccomponents was analyzed through one-way repeatedmeasures analysis of variance (RM-ANOVA) for timecourse changes or 2-way RM-ANOVA for effects on the group, time, and group-by-time interaction. Analyses were followed by the post hoc Student-Newman-Keuls test (SigmaPlot v.11, Systat Software, Chicago, IL). Behavioral and immunostaining data were analyzed using one-way ANOVA, followed by a post hoc Tukey test. A $P$ value less than 0.05 was considered to be a significant difference.

\section{Results}

\section{In Vivo EP Recording in the Spinal Dorsal Cord}

The application of electric conditioning stimulation evidently evoked 2 negative potentials, A-component (0 - $90 \mathrm{~ms}$ ) and C-component (90 - $300 \mathrm{~ms})$ (Fig. 1A). Nissl staining of all the tested spinal cords showed the recording sites, and only those with a lesion limited to the superficial laminae were qualified (Fig. 1B).

\section{PRF Differentially Suppressed the C-component in Normal Rats}

PRF differentially affected the EPs of the A- and C-components in the normal animals. Figure $2 \mathrm{~A}$ shows the recording traces in the sham and PRF groups at various time points. Figures $2 \mathrm{~B}$ and $2 \mathrm{C}$ display the response ratio of the 2 components, respectively. In the sham-PRF group (Sham), both the A- and C-components remained consistent without time-course changes compared with the baseline value at 0 minutes for at least 120 minutes (except for 100 and 120 minutes in the PRF group; $P=$ .813 for the A-component in Fig. $2 \mathrm{~B} ; P=.277$ for the C-component in Fig. 2C). In the PRF group (PRF), the A-component was gradually enhanced with high variations, but no significant difference was discerned over time ( $P=.123$, Fig. 2B). PRF significantly suppressed the $C$-component over time $(P=.001$; Fig. $2 C)$ after 30 minutes. 
We performed group comparisons in the A- and C-component, respectively. For the A-component (Fig. 2B), a significant interaction between the treatment and time was found through 2-way RM-ANOVA $(P=$ .040), and the effect was dependent on time. By contrast, no significant interaction was discerned between the treatment and time for the C-component $(P=.153$; Fig. 2C). The results revealed that PRF exerted differential effects on A-versus C-fiber-mediated EPs.

\section{PRF Differentially Suppressed the C-component, but not the A-component, in SNL Rats}

Similar to results in normal rats, PRF differentially modulated the A-/C-components (Fig. 3A) under neuropathic pain. In the sham group (SNL+Sham), both the Aand $\mathrm{C}$-components remained constant over 120 minutes $(P=.499$ for the A-component in Fig. 3B; $P=.99$ for the C-component in Fig. $3 C$ ). In the PRF rats (SNL+PRF), the C-component was significantly depressed at all time points after treatment $(P=.001$; Fig. 3C), whereas the A-component exhibited high variations (Fig. 3B) without significant time-course changes ( $P=.056$; Fig. 3B).

Next, the C-component was significantly depressed in the SNL+PRF group compared with the SNL+sham group during $80-120$ minutes (Fig. 3C). A significant interaction was present between groups for C-components $(P=.004$; Fig. $3 \mathrm{C})$. However, for A-components, no significant interaction was found between the treatment and time ( $P=1.000$; Fig 3B). The results revealed that PRF produced a selective inhibitory effect on C-fiber-mediated EPs in SNL rats, but could not affect A-fiber-mediated EPs.

\section{PRF Inhibited Rapid p-ERK Induction by Mechanical Nociception in the Control and SNL Rats}

To mimic C-components induced by suprathreshold nerve stimuli, intense mechanical stimulations (MS) with a thick von Frey fiber were applied. We determined that repetitive MS in the control rats engendered an increased expression of $\mathrm{p}$-ERK in the superficial laminae I and II at 30 minutes, but few p-ERK-ir cells in the deeper laminae III-V (the C+MS+Sham in Figs. 4A and $4 B)$. PRF treatment significantly reduced number of the p-ERK-ir cells in the superficial laminae both at L5, the level at which the DRG was stimulated, and L4 (Fig. 4B). PRF per se did not increase p-ERK compared with sham treatment ( $C+$ PRF vs. $C+$ Sham, $P>0.05)$ at 30 minutes poststimulation (Fig. 4B).

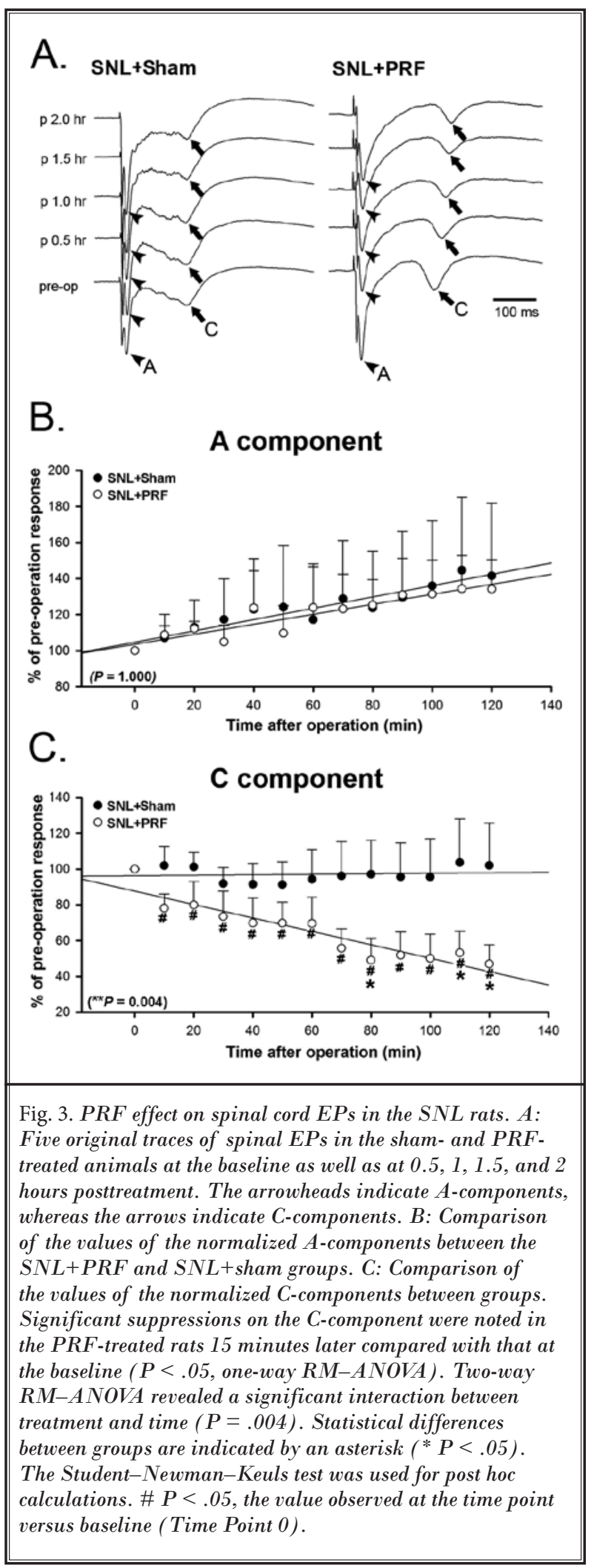




\section{A. Post-PRF 30 min}
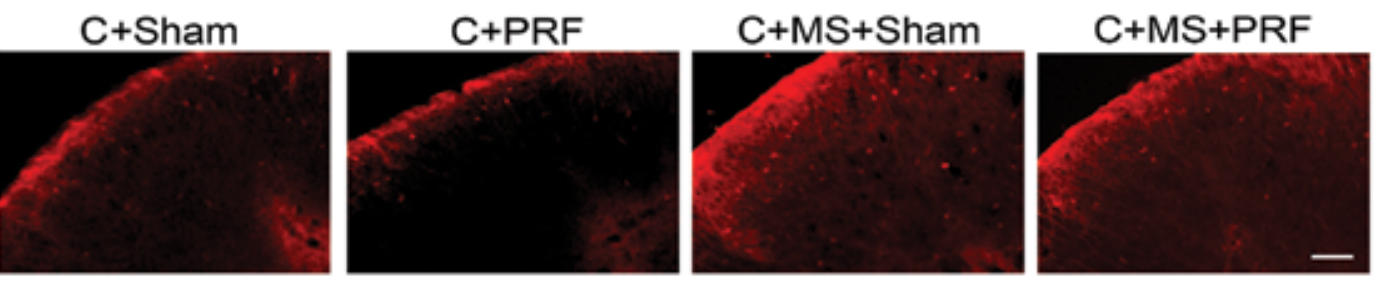

\section{B. Post-PRF 30 min}
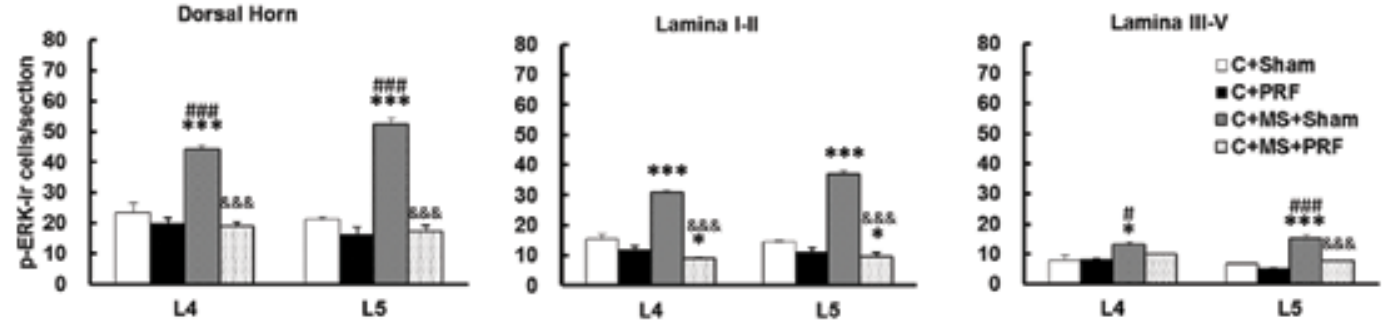

\section{Post-PRF 30 min}
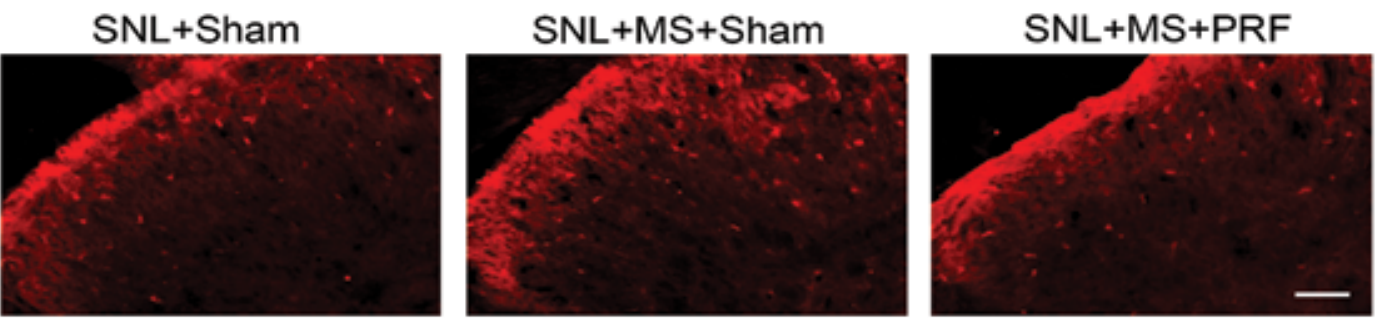

\section{Post-PRF 30 min}
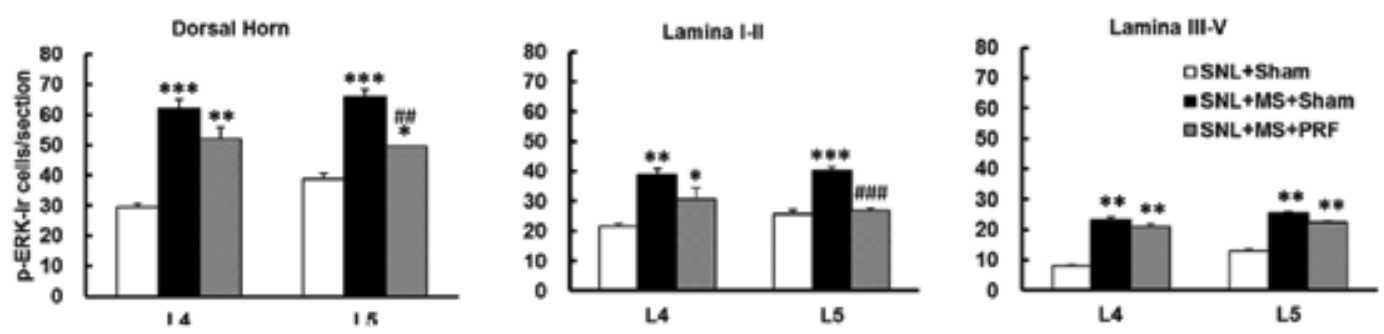

Fig. 4. PRF effect on early $p$-ERK expression evoked by mechanical noxious stimulation (MS) in the control (C) and $S N L$ rats. A: Immunofluorescent staining revealed p-ERK inductions in the control rats that received the sham, PRF, repetitive MS, or MS+PRF ( $C+$ Sham, $C+P R F, C+M S+$ Sham, $C+M S+P R F$, Scale bar: $100 \mu m)$. B: Analysis of p-ERK-immunoreactive cell numbers in these groups. C: Immunofluorescence showed p-ERK expression in the SNL rats that received the sham PRF, repetitive MS, and MS+PRF (SNL+Sham, SNL+MS+Sham, SNL+MS+PRF; Scale bar: $100 \mu m$ ). D: Analysis of p-ERKimmunoreactive cell numbers in these groups. One-way ANOVA with a post hoc Tukey test.

${ }^{*} \mathrm{P}<.05,{ }^{* *} \mathrm{P}<.01{ }^{* * *} \mathrm{P}<.001$ for groups versus $\mathrm{C}+$ Sham or $\mathrm{SNL}+\mathrm{Sham}$; $\mathrm{P}<.05$, \#\# $\mathrm{P}<.01$, \#\#\# $\mathrm{P}<.001$ for groups versus $\mathrm{C}+\mathrm{PRF}$ or $\mathrm{SNL}+\mathrm{MS}+\mathrm{Sham} ; \& \& \& \mathrm{P}<.001$ for $\mathrm{C}+\mathrm{MS}+\mathrm{PRF}$ versus $\mathrm{C}+\mathrm{MS}+\mathrm{Sham}$. 
In SNL rats, MS significantly increased p-ERK in the SNL-sensitized spinal cord, compared with those in the non-MS rats (SNL+MS+Sham vs. SNL+Sham, Figs. 4C and 4D). PRF inhibited p-ERK only in the L5 superficial laminae, but had no effect in the deeper laminae (SNL+MS+PRF vs. SNL+MS+Sham; Fig. 4D). Altogether, PRF rapidly mitigated newly induced $p$-ERK in both control and hypersensitive spinal cords, whereas did not induce $p$-ERK by itself.

\section{PRF Attenuated SNL-induced Nociceptive Hypersensitivity for a Prolonged Period}

SNL resulted in drastically mechanical and thermal hypersensitivities (SNL+Sham group in Figs. 5A and 5B; $P=.000$ for most post-SNL data vs baseline data; and $P$ $<.001$ for SNL+Sham vs C+Sham, not shown). PRF significantly suppressed tactile allodynia for 10 days $(P<.005$ at PD10; Fig. 5A) and reduced thermal hyperalgesia for 2 weeks ( $P<.01$ at PD14; Fig. 5B). Collectively, PRF produced a prolonged analgesic effect on SNL-induced pain.

\section{PRF Reduced SNL-induced and Maintained Late ERK Activations in Spinal Dorsal Horns}

Figure 6 shows the delayed effects of PRF on p-ERK expression on post-PRF Day 1 and Day 14 (i.e., PD1 and PD14, Fig. 5). SNL increased p-ERK-ir cells to a similar level in the superficial laminae at both time points (Figs. $6 \mathrm{~B}$ and $6 \mathrm{C})$, whereas it caused more p-ERK-ir cells in the deeper laminae (III-V) on PD14 (Fig. 6C). PRF (SNL+PRF) reduced the number of $p$-ERK-ir cells in both the L4-L5 superficial and L5 deep laminae compared with those in the SNL+Sham group at both time points $(P<.05$; Figs. $6 \mathrm{~B}$ and $6 \mathrm{C}$ ), indicating a persistent inhibitory effect. Double staining on PD14 (Fig. 6D) revealed most pERK-ir cells were neurons (NeuN) and a few others were astrocytes (GFAP), but none were microglia (OX42).

\section{PRF Does Not Change Baseline Withdrawal Thresholds}

We further examined if PRF alters normal rats (Fig. 7). All PRF-treated rats were found to exhibit normal movements, without limping or unbalanced walking at the hind limbs, for 3 postoperative days. The withdrawal thresholds did not differ, either between both hind paws in the same group (i.e., PRF-L vs PRF-R) or the same left paws between groups (i.e., PRF-L vs Sham-L) at all time points. The mild threshold drops over the left paws in both groups may be attributed to surgery-related pain, but no statistical significance was obtained. Thus, neither the electrode placement nor PRF current

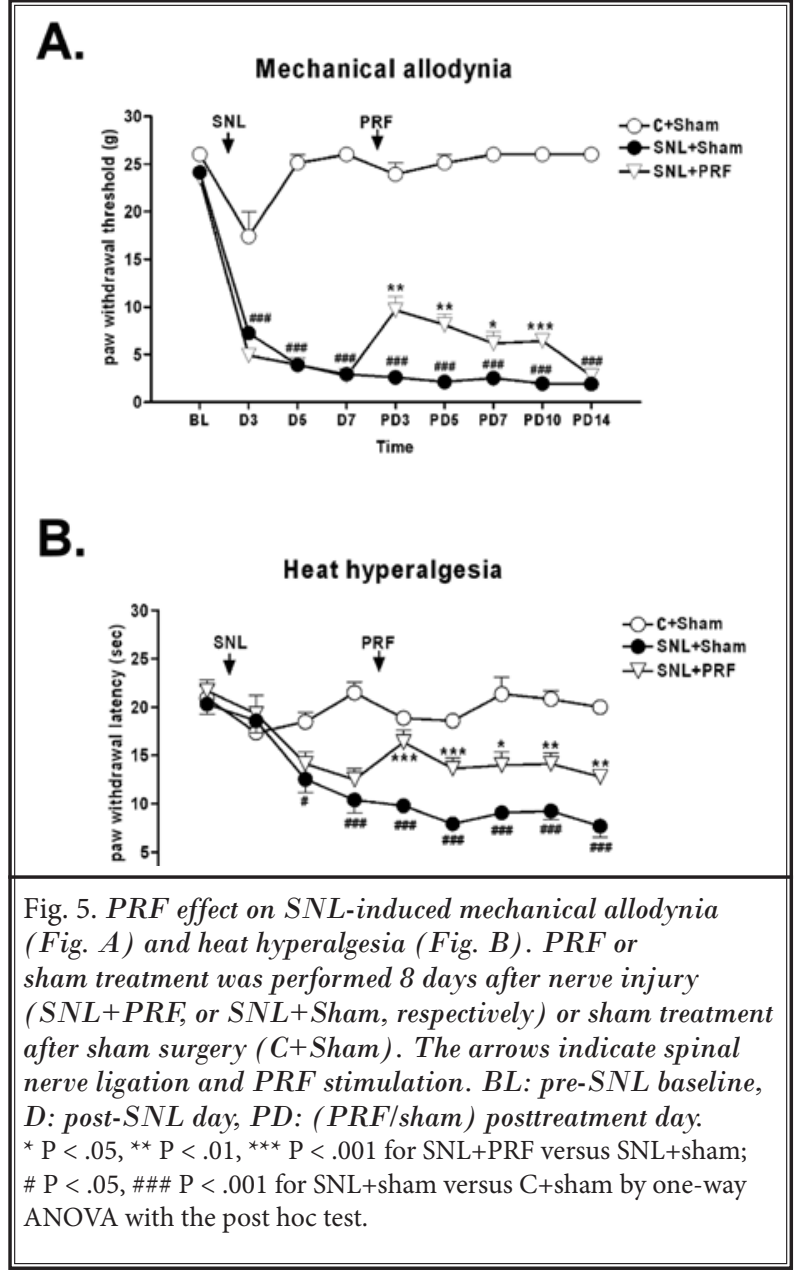

could alter basal sensory thresholds and behaviors.

\section{PRF May Not Injure DRG Neurons}

ATF-3 expression in the nuclei of DRG neurons suggests peripheral nerve injury. Weak ATF-3 staining was noted in the left L4 and L5 DRG neurons in the Sham, Sham-E, and PRF groups (panels a, b, and c, respectively, in Figs. 8A and 8B). Little to no ATF-3 was found in the contralateral DRGs (panel d, Figs. 8A and 8B). The expressive ratio of ATF-3 neurons among the Sham, Sham$\mathrm{E}$, and PRF groups yielded no statistical differences $(P>$ .05 , Fig. 8C). Cell-size distribution analysis revealed that most of the ATF-3-ir cells were small neurons, presumably unmyelinated C-fiber-neurons ( $<400 \mu \mathrm{m} 2$, Fig. 8D). However, no difference was observed among the groups in terms of cell-size distribution. Accordingly, neither the electrode placement nor PRF stimulation induced ATF-3 expression. 


\section{A. Post-PRF 1 Day}
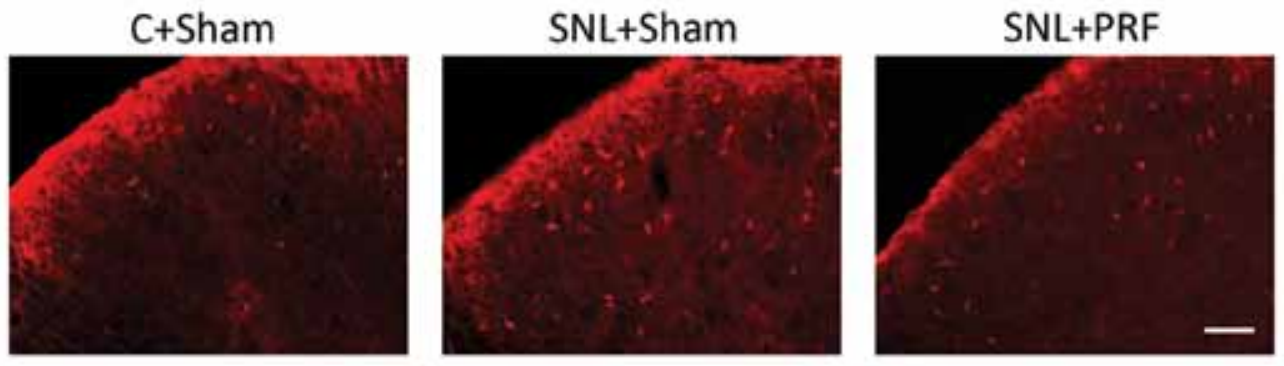

\section{B. Post-PRF 1 Day}
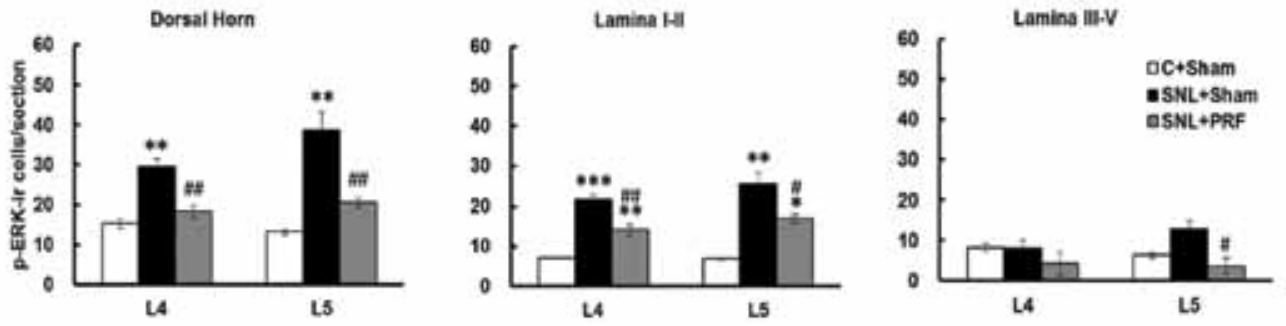

\section{Post-PRF 14 Day}
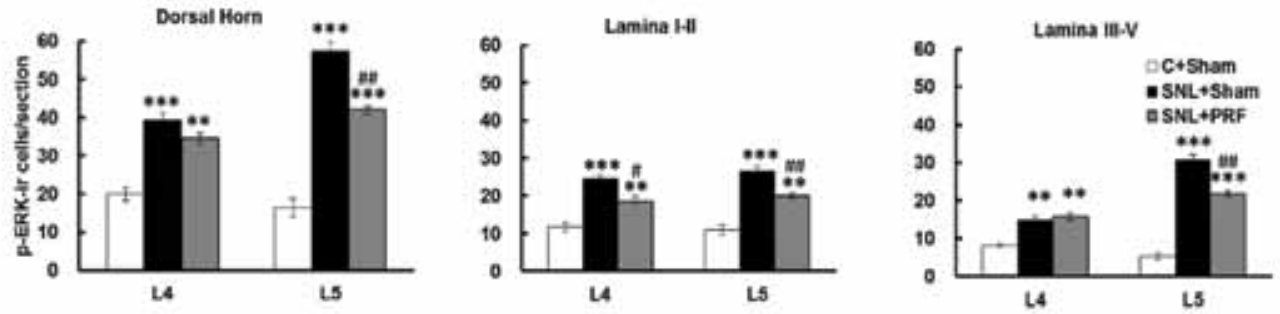

\section{D. p-ERK expression}
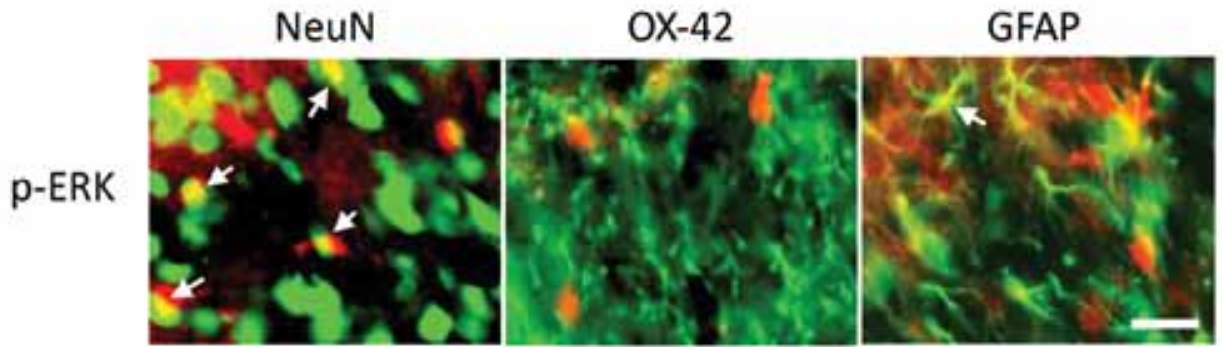

Fig. 6. PRF effect on SNL-induced late ERK activation in the spinal dorsal horn. A: Expression of p-ERK in the control rats with sham-PRF (C+Sham), in the SNL rats with sham-PRF (SNL+sham), and in the SNL rats with PRF (SNL+PRF) at one day post-PRF. Scale bar $=100 \mu \mathrm{m}$. B: Comparison of $p$-ERK-immunoreactive cell numbers among the groups at one day postPRF; C: p-ERK comparison at 14 days post-PRF. One-way ANOVA with post hoc Tukey's test.

${ }^{\star} \mathrm{P}<.05,{ }^{* *} \mathrm{P}<.01,{ }^{* * *} \mathrm{P}<.001$ for groups versus $\mathrm{C}+$ Sham; \# $\mathrm{P}<.05$, \#\# $\mathrm{P}<.01$ for SNL+PRF versus SNL+Sham. D: Double staining of $\mathrm{p}-\mathrm{ERK}$ with neuronal, microglial, and astrocytic markers (NeuN, OX42, and GFAP, respectively). The arrowheads indicate colocalization. Scale bar $=$ $10 \mu \mathrm{m}$. 


\section{Discussion}

We demonstrated that bipolar PRF treatment persistently suppressed SNL-induced and SNL-maintained nociceptive hypersensitivity with electrophysiological, molecular, and behavioral evidence. In particular, we identified a selective long-term depression (LTD) of PRF on C-fiber-mediated spinal EPs and ERK activations, which determines chronic pain neuromodulation.

\section{PRF Selectively Suppresses C fiber-mediated EPs}

The major finding that PRF selectively suppresses the C-component is in agreement with empirical observations that PRF affects only pain sensations, but not innocuous sensations or motor functions. In addition to our EP results, physical laws support the phenomenon.

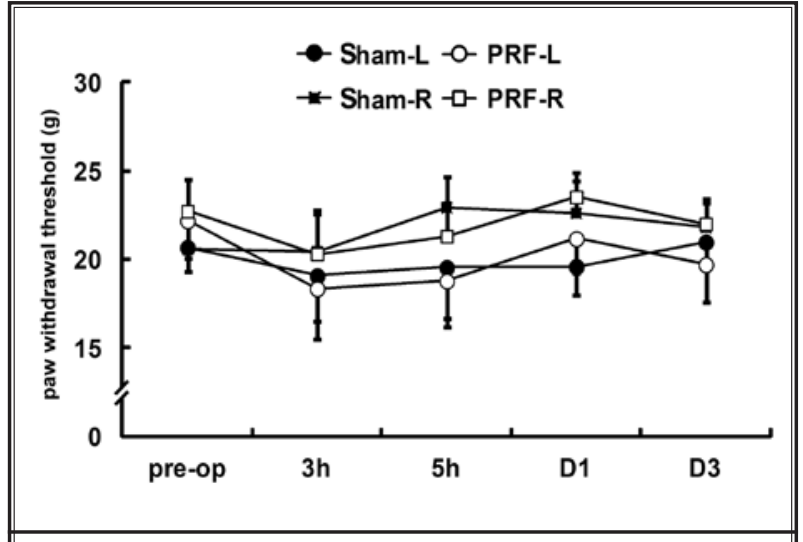

Fig. 7. PRF treatment effect on withdrawal thresholds at the bilateral hind paws in the normal rats. Sham-L/ Sham-R: left/right hind paw of the sham-PRF rats; PRF-L/ PRF-R: left/right hind paw of the PRF-treated rats.

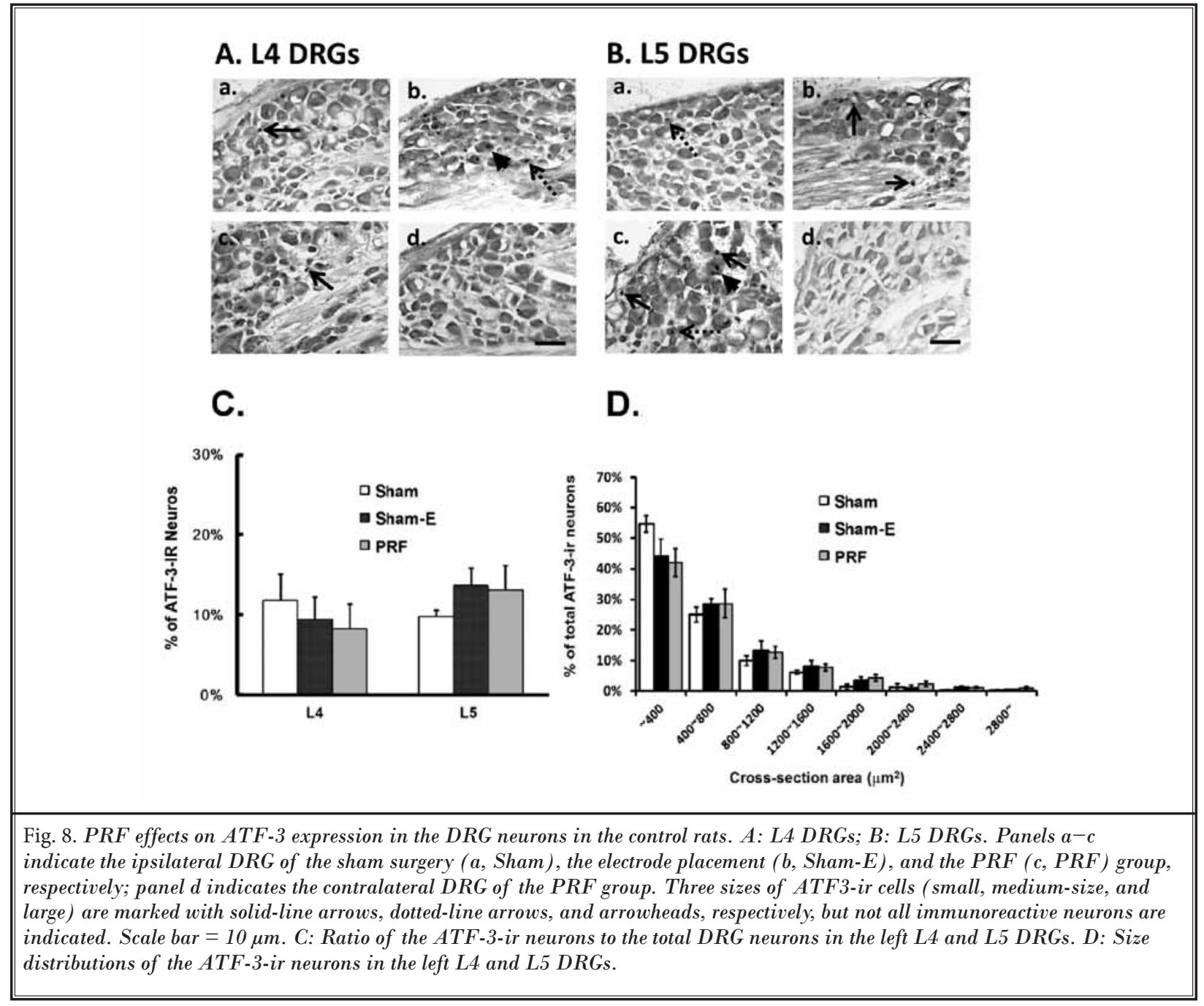


Though PRF can exert both thermal field (T-field) and electric field (E-field) effects, it generates stronger Efield and transmembrane potentials in neurons, but much lower T-field and temperature in comparison with CRF (8). E-field of PRF causes various degrees of microinjury in DRG neurons $(10,12-15,39)$, but most damage is very mild and reversible within 4 weeks (40). Since E-field gradient across neural membrane is influenced by numerous factors such as the thickness of the myelin sheet, unmyelinated C-fibers may be vulnerable to Efield exposure (14). PRF was reported to produce higher axonal damage in $\mathrm{C}$-fibers than in $A \beta$ and $A \delta$ fibers of the sciatic nerve (14) to yield a selective blockade.

\section{Early PRF Effects: LTD on the C-component and P-ERK}

We demonstrated that 5-minute PRF produced LTD on C-fiber-mediated EPs for at least 2 hours in both control and SNL rats without affecting A-fiber-mediated EPs. Similar electrostimulation-induced synaptic modulations were reported in the brain, DRG, and cutaneous tissue. For instance, low-frequency electric pulses $(1-\mathrm{Hz}$, 900 seconds) at the DRG produced LTD in A $\delta$ and C-fiber synapses for 2 hours (41), and high-frequency tetanic stimulation (100- $\mathrm{Hz}$, rapid 3 repeats) induced LTD in the spinal motor withdrawal (21). Although electric parameters were inconclusive among studies, low-frequency stimulation seems to have better chance to produce LTD (8). Moreover, compared with an in vitro study conducting PRF in hippocampal slices to obtain a less than 15-minute inhibition of excitatory postsynaptic amplitudes (7), the depressive duration in this in vivo study is much longer. The intact and complex circuitry, in contrast with monosynaptic connections in the slices, may contribute to the prolonged duration.

Akin to the immediateness in EP recordings, p-ERK appears rapidly and exclusively in superficial dorsal neurons after strong von Frey stimuli in both normal and SNL rats. Our results are consistent with studies showing p-ERK appears 15 minutes after a peripheral electric tetanus or 2 minutes after intense mechanical stimulation (35). Because activation of neuronal ERKCREB pathway is attributed to early spinal LTP (24), the reduction of stimulation-evoked p-ERK supports LTD in electrophysiological findings. Of particular note, expression of p-ERK in the SNL rats (Fig. 4C and 4D) includes both SNL-maintained $p$-ERK and von-Freyinduced new $p$-ERK. The final $p$-ERK inhibition indicates an integrated effect on neuropathic and nociceptic signals.

\section{Delayed PRF Effects: Prolonged Analgesia and p-ERK Inhibition in Neurons and Glia}

In a well-developed SNL-maintained pain, a single PRF treatment reduced mechanical- and heat-evoked nociception for 10 and 14 days, respectively. The result lasted longer than that obtained in our previous study, which showed effects for 5 days and 7 days, respectively (27). It implies that PRF may exert better effect on the late-stage pain than on the early-stage pain. Nerve injury could also sensitize uninjured medium-sized/large neurons or axons and alter their functions from relaying innocuous transmission to nociceptive information, namely a cell-type shift $(42,43)$. Hypothetically, a higher proportion of C-fiber-mimic hypersensitivity would be present in the affected DRG over a period of time and exhibits higher susceptibility to PRF treatment.

p-ERK is critical in spinal sensitization both in initiating nociceptive development and maintaining neuroplasticity $(25,44)$. PRF attenuated spinal p-ERK at 30 minutes, one day, and even 2 weeks, which outlasts its effect on pain behaviors. Moreover, p-ERK inhibition is neither limited to L5 (the stimulated DRG level) nor restrictive in superficial SDH at 14 days post-PRF (Fig. 6C). The above findings suggest PRF produces long-lasting and widespread effects, and also answers a persistent Fos induction in the cervical SDH at 3 hours and 7 days post-PRF $(16,45)$.

Astrocytic p-ERK expression needs particular notice. Spinal glia sustain late-phase LTP in neuropathic pain for days, weeks, or even longer (25). Inhibition of glial activity could convert tetanus-induced postsynaptic potentials from LTP to LTD $(46,47)$, indicating a neural-glial crosstalk (48). We previously determined that PRF inhibited SNL-induced microglial p-p38 and TNF (27). In a CFA-induced pain study, PRF suppressed spinal p-JNK for 14 days (49), and CFA-induced pJNK \{sp?\} was found to be exclusively expressed in astrocytes (50). Meanwhile, PRF may activate the expression of several types of cytokines (40). Overall, PRF application can directly and indirectly modulate neuron-glia-mediated spinal sensitization and neuroinflammation.

\section{PRF Does Not Lead to Neuronal Stress in the DRG}

Neither the PRF current nor electrode insertion induced neuronal stress in the treated DRG. ATF-3 is a member of the ATF/CREB transcription factor superfamily, and indicates stresses in DRG neurons after peripheral axonal injury or nonneural tissues following cellular destruction (51-53). ATF-3 was also suggested to 
be involved in nociceptive development (54). Because the active zone of PRF was limited to a narrow range adjacent to the electrode tip $(7,8,11)$, the severely affected tissue is theoretically the region in close contact with the electrode. However, no dense ATF-3-gathering region was observed in the DRG sections, nor was any significant difference in the ATF-3 expression ratio or cell-size distribution noted among groups. Our results are distinct from those obtained by Hamann et al (55), who reported a small but significant increase in ATF-3 expression in the DRGs between the PRF and control groups. However, the voltage was much lower in our study than that study, so E-field effects may be insufficient to induce damage. A potential thermal injury by heat spikes (8) at the electrode tip can be negligible based on our measurement before (29). We thus conclude that low-volt bipolar PRF is safe for the DRG under our study profile.

\section{Limitations}

Translation of our animal data to clinical applications needs to be done very carefully. First, the SNL model elicits distinct phenotypic changes which cannot represent all neuropathic pain conditions (56); second, there are disparities in pain measurements between animal models using withdrawal responses and human studies with spontaneous perception (57); third, molecular changes in the injured DRG were not investigated, i.e., ATF-3 and p-ERK. It is important to investigate how the DRG was reversed by PRF. Besides, it is completely unknown if such a bipolar PRF application causes any unpleasant sensation in rats and in humans.

\section{Conclusion}

In this study, we reveal that PRF produces a prolonged analgesic effect with minimal neural injury. It induces spinal LTD to selectively modulate C-fiber transmission, inhibits postsynaptic excitability and sequential ERK activations, and alleviates pain. Our findings support PRF treatment as a valuable intervention therapy for chronic neuropathic pain.

\section{Acknowledgment}

This study was sponsored by research grants from National Science Council in Taiwan (NSC99-2628-B341-001-MY2, NSC101-2314-B-039-005-MY3), National Research Program for Biopharmaceuticals (DOH101-TDPB-111-NSC006), in part from Taiwan Ministry of Health and Welfare Clinical Trial and Research Center of Excellence (MOHW105-TDU-B-212-133019) and China Medical University under the Aim for Top University Plan of the Ministry of Education in Taiwan to Y.-R. Wen, and from National Science Council in Taiwan (NSC 99-2321B-002-034) to C.-W. Lin

\section{References}

1. Bogduk N. Pulsed radiofrequency. Pain Med 2006; 7:396-407.

2. Byrd D, Mackey S. Pulsed radiofrequency for chronic pain. Curr Pain Headache Rep 2008; 12:37-41.

3. Van Boxem K, Cheng J, Patijn J, van Kleef M, Lataster A, Mekhail N, Van Zundert J. 11. Lumbosacral radicular pain. Pain Pract 2010; 10:339-358.

4. Chua NH, Vissers KC, Sluijter ME. Pulsed radiofrequency treatment in interventional pain management: Mechanisms and potential indicationsa review. Acta Neurochir (Wien) 2011; 153:763-771.

5. Cahana A, Van Zundert J, Macrea L, van Kleef $M$, Sluijter $M$. Pulsed radiofrequency: Current clinical and biological literature available. Pain Med 2006; 7:411-423.

6. Sluijter ME, Cosman ER, Rittman IWB, van Kleef $M$. The effects of pulsed ra- diofrequency field applied to the dorsal root ganglion: A preliminary report. Pain Clinic 1998; 11:109-117.

7. Cahana A, Vutskits L, Muller D. Acute differential modulation of synaptic transmission and cell survival during exposure to pulsed and continuous radiofrequency energy.J Pain 2003; 4:197-202.

8. Cosman ER, Jr., Cosman ER, Sr. Electric and thermal field effects in tissue around radiofrequency electrodes. Pain Med 2005; 6:405-424.

9. Cosman ER, Nashold BS, Ovelman-Levitt $J$. Theoretical aspects of radiofrequency lesions in the dorsal root entry zone. Neurosurgery 1984; 15:945-950.

10. Erdine S, Yucel A, Cimen A, Aydin S, Sav A, Bilir A. Effects of pulsed versus conventional radiofrequency current on rabbit dorsal root ganglion morphology. Eur J Pain 2005; 9:251-256.

11. Sluijter M, Racz G. Technical aspects of radiofrequency. Pain Pract 2002; 2:195-200.

12. Protasoni $M$, Reguzzoni $M$, Sangiorgi S, Reverberi C, Borsani E, Rodella LF, Dario A, Tomei G, Dell'Orbo C. Pulsed radiofrequency effects on the lumbar ganglion of the rat dorsal root: A morphological light and transmission electron microscopy study at acute stage. Eur Spine J 2009; 18:473-478.

13. Tun K, Cemil B, Gurcay AG, Kaptanoglu E, Sargon MF, Tekdemir I, Comert A, Kanpolat Y. Ultrastructural evaluation of pulsed radiofrequency and conventional radiofrequency lesions in rat sciatic nerve. Surg Neurol 2009; 72:496-500.

14. Erdine S, Bilir A, Cosman ER, Cosman $E R, J$ r. Ultrastructural changes in axons following exposure to pulsed radiofrequency fields. Pain Pract 2009; 9:407-417.

15. Tun K, Savas A, Sargon MF, Solaroglu I, Kanpolat Y. The histopathological and electron-microscopic examination of 
the stereotactic pulsed radiofrequency and conventional radiofrequency thermocoagulation lesions in rat brain. $\mathrm{Neu}-$ rol Res 2006; 28:841-844.

16. Van Zundert J, de Louw AJ, Joosten EA, Kessels AG, Honig W, Dederen PJ, Veening JG, Vles JS, van Kleef M. Pulsed and continuous radiofrequency current adjacent to the cervical dorsal root ganglion of the rat induces late cellular activity in the dorsal horn. Anesthesiology 2005: 102:125-131.

17. Yang $\mathrm{CH}$, Chen $\mathrm{KH}$, Huang HW, SheenChen SM, Lin CR. Pulsed radiofrequency treatment attenuates increases in spinal excitatory amino acid release in rats with adjuvant-induced mechanical allodynia. Neuroreport 2013; 24:431-436.

18. Hagiwara S, Iwasaka H, Takeshima N, Noguchi T. Mechanisms of analgesic action of pulsed radiofrequency on adjuvant-induced pain in the rat: Roles of descending adrenergic and serotonergic systems. Eur J Pain 2009; 13:249-252.

19. Sandkuhler J. Understanding LTP in pain pathways. Mol Pain 2007; 3:9.

20. Sandkuhler J, Gruber-Schoffnegger D. Hyperalgesia by synaptic long-term potentiation (LTP): An update. Current Opinion in Pharmacology 2012; 12:18-27.

21. You HJ, Tjolsen A, Arendt-Nielsen L. High-frequency conditioning electrical stimulation evokes supraspinal independent long-term depression but not long-term potentiation of the spinal withdrawal reflex in rats. Brain Research 2006; 1090:116-122.

22. Pfau DB, Klein T, Putzer D, PogatzkiZahn EM, Treede RD, Magerl W. Analysis of hyperalgesia time courses in humans after painful electrical high-frequency stimulation identifies a possible transition from early to late LTP-like pain plasticity. Pain 2011; 152:1532-1539.

23. Chen $T$, O'Den $G$, Song $Q$, Koga $K$, Zhang MM, Zhuo M. Adenylyl cyclase subtype 1 is essential for late-phase long term potentiation and spatial propagation of synaptic responses in the anterior cingulate cortex of adult mice. Molecular Pain 2014; 10:65.

24. Xin WJ, Gong QJ, Xu JT, Yang HW, Zang Y, Zhang T, Li YY, Liu XG. Role of phosphorylation of ERK in induction and maintenance of LTP of the C-fiber evoked field potentials in spinal dorsal horn. Journal of Neuroscience Research 2006; 84:934-943.

25. Zhou LJ, Zhong Y, Ren WJ, Li YY, Zhang
T, Liu XG. BDNF induces late-phase LTP of $\mathrm{C}$-fiber evoked field potentials in rat spinal dorsal horn. Experimental Neurology 2008; 212:507-514.

26. Ruscheweyh R, Wilder-Smith O, Drdla R, Liu XG, Sandkuhler J. Long-term potentiation in spinal nociceptive pathways as a novel target for pain therapy. Mol Pain 2011; 7:20.

27. Lin ML, Lin WT, Huang RY, Chen TC, Huang SH, Chang CH, Tsai SY, Chiu HW, Yeh GC, Lin CW, Wen YR. Pulse radiofrequency inhibited activation of spinal mitogen-activated protein kinases and ameliorated early neuropathic pain in rats. Eur J Pain 2014; 18: 659-670.

28. Zimmermann M. Ethical guidelines for investigations of experimental pain in conscious animals. Pain 1983; 16:109-110.

29. Chiu HW, Lin ML, Lin CW, Lin WT, Ho $\mathrm{IH}$, Fang PH, Wen YR, Lu SS. Pain control on demand based on pulsed radiofrequency stimulation of the dorsal root ganglion using a batteryless implantable CMOS SoC. IEEE Trans Biom Circuits Syst 2010; 4:350-359.

30. Kim SH, Chung JM. An experimental model for peripheral neuropathy produced by segmental spinal nerve ligation in the rat. Pain 1992; 50:355-363.

31. Li Y, Dorsi MJ, Meyer RA, Belzberg AJ. Mechanical hyperalgesia after an $L_{5}$ spinal nerve lesion in the rat is not dependent on input from injured nerve fibers. Pain 2000; 85:493-502.

32. Chaplan SR, Bach FW, Pogrel JW, Chung JM, Yaksh TL. Quantitative assessment of tactile allodynia in the rat paw. J Neurosci Methods 1994; 53:55-63.

33. Dixon WJ. Efficient analysis of experimental observations. Annual Review of Pharmacology and Toxicology 1980; 20:441-462.

34. Thompson SW, Woolf CJ, Sivilotti LG. Small-caliber afferent inputs produce a heterosynaptic facilitation of the synaptic responses evoked by primary afferent A-fibers in the neonatal rat spinal cord in vitro. Journal of Neurophysiology 1993; 69:2116-2128.

35. Ji RR, Baba H, Brenner GJ, Woolf CJ. Nociceptive-specific activation of ERK in spinal neurons contributes to pain hypersensitivity. Nat Neurosci 1999; 2:1114-1119.

36. Mizukoshi K, Sasaki M, Izumi Y, Miura M, Watanabe M, Amaya F. Activation of p38 mitogen-activated protein kinase in the dorsal root ganglion contributes to pain hypersensitivity after plantar incision. Neuroscience 2013; 234C:77-87.

37. Obata K, Yamanaka H, Dai Y, Mizushima T, Fukuoka T, Tokunaga A, Noguchi K. Differential activation of MAPK in injured and uninjured DRG neurons following chronic constriction injury of the sciatic nerve in rats. Eur J Neurosci 2004; 20:2881-2895.

38. Obata K, Yamanaka H, Kobayashi K, Dai Y, Mizushima T, Katsura H, Fukuoka T, Tokunaga A, Noguchi K. Role of mitogen-activated protein kinase activation in injured and intact primary afferent neurons for mechanical and heat hypersensitivity after spinal nerve ligation. J Neurosci 2004; 24:10211-10222.

39. Podhajsky RJ, Sekiguchi Y, Kikuchi S, Myers RR. The histologic effects of pulsed and continuous radiofrequency lesions at 42 degrees $C$ to rat dorsal root ganglion and sciatic nerve. Spine (Phila Pa 1976) 2005; 30:1008-1013.

40. Choi S, Choi HJ, Cheong Y, Chung SH, Park HK, Lim YJ. Inflammatory responses and morphological changes of radiofrequency-induced rat sciatic nerve fibres. Eur J Pain 2014; 18:192-203.

41. Sandkuhler J, Chen JG, Cheng G, Randic M. Low-frequency stimulation of afferent Adelta-fibers induces long-term depression at primary afferent synapses with substantia gelatinosa neurons in the rat. J Neurosci 1997; 17:6483-6491.

42. Ruscheweyh R, Forsthuber L, Schoffnegger D, Sandkuhler J. Modification of classical neurochemical markers in identified primary afferent neurons with Abeta-, Adelta-, and C-fibers after chronic constriction injury in mice. The Journal of Comparative Neurology 2007; 502:325-336.

43. Djouhri L, Fang X, Koutsikou S, Lawson SN. Partial nerve injury induces electrophysiological changes in conducting (uninjured) nociceptive and nonnociceptive DRG neurons: Possible relationships to aspects of peripheral neuropathic pain and paresthesias. Pain 2012; 153:1824-1836.

44. Zhuang ZY, Gerner P, Woolf CJ, Ji RR. ERK is sequentially activated in neurons, microglia, and astrocytes by spinal nerve ligation and contributes to mechanical allodynia in this neuropathic pain model. Pain 2005; 114:149-159.

45. Higuchi Y, Nashold BS, Jr., Sluijter M, 
Cosman E, Pearlstein RD. Exposure of the dorsal root ganglion in rats to pulsed radiofrequency currents activates dorsal horn lamina I and II neurons. Neurosurgery 2002; 50:850-855; discussion 856 .

46. Gruber-Schoffnegger D, Drdla-Schutting R, Honigsperger C, Wunderbaldinger G, Gassner M, Sandkuhler J. Induction of thermal hyperalgesia and synaptic long-term potentiation in the spinal cord lamina I by TNF-alpha and IL-ibeta is mediated by glial cells. J Neurosci 2013; 33:6540-6551.

47. Ma JY, Zhao ZQ. The involvement of glia in long-term plasticity in the spinal dorsal horn of the rat. Neuroreport 2002; 13:1781-1784.

48. Edelmayer RM, Brederson JD, Jarvis MF, Bitner RS. Biochemical and pharmacological assessment of MAP-kinase signaling along pain pathways in experimental rodent models: A potential too for the discovery of novel antinociceptive therapeutics. Biochem Pharmacol 2014; 87:390-398.

49. Chen $\mathrm{KH}$, Yang $\mathrm{CH}$, Juang $\mathrm{SE}$, Huang HW, Cheng JK, Sheen-Chen SM, Cheng
JT, Lin CR. Pulsed radiofrequency reduced complete Freund's adjuvant-induced mechanical hyperalgesia via the spinal c-Jun $\mathrm{N}$-terminal kinase pathway. Cellular and Molecular Neurobiology 2014; 34:195-203.

50. Gao YJ, Xu ZZ, Liu YC, Wen YR, Decosterd I, Ji RR. The c-Jun N-terminal kinase 1 (JNK1) in spinal astrocytes is required for the maintenance of bilateral mechanical allodynia under a persistent inflammatory pain condition. Pain 2010; 148:309-319.

51. Tsujino $\mathrm{H}$, Kondo E, Fukuoka $\mathrm{T}$, Dai $\mathrm{Y}$, Tokunaga A, Miki K, Yonenobu K, Ochi $T$, Noguchi K. Activating transcription factor 3 (ATF3) induction by axotomy in sensory and motoneurons: A novel neuronal marker of nerve injury. Mol Cell Neurosci 2000; 15:170-182.

52. Seijffers R, Allchorne AJ, Woolf CJ. The transcription factor ATF-3 promotes neurite outgrowth. Mol Cell Neurosci 2006; 32:143-154.

53. Hartman MG, Lu D, Kim ML, Kociba G), Shukri T, Buteau J, Wang X, Frankel WL, Guttridge D, Prentki M, Grey
ST, Ron D, Hai T. Role for activating transcription factor 3 in stress-induced beta-cell apoptosis. Mol Cell Biol 2004; 24:5721-5732.

54. Hirose K, Iwakura N, Orita S, Yamashita $M$, Inoue G, Yamauchi K, Eguchi Y, Ochiai N, Kishida S, Nakamura J, Takaso $M$, Ishikawa $T$, Arai G, Miyagi $M$, Kamoda H, Aoki Y, Hiwatari R, Kakizaki J, Kunishi T, Kono M, Suzuki T, Toyone T, Takahashi K, Kuniyoshi K, Ohtori S. Evaluation of behavior and neuropeptide markers of pain in a simple, sciatic nerve-pinch pain model in rats. Eur Spine J 2010; 19:1746-1752.

55. Hamann W, Abou-Sherif S, Thompson $S$, Hall S. Pulsed radiofrequency applied to dorsal root ganglia causes a selective increase in $\mathrm{ATF}_{3}$ in small neurons. Eur J Pain 2006; 10:171-176.

56. Challa SR. Surgical animal models of neuropathic pain: Pros and cons. Int J Neurosci 2015; 125:170-174.

57. Vierck CJ, Yezierski RP. Comparison of operant escape and reflex tests of nociceptive sensitivity. Neurosci Biobehav Rev 2015; 51:223-242. 
